\title{
A Modified Method of Forecasting Enrollments Based on Fuzzy Time Series
}

\author{
Hao Feng \\ College of Science and Engineering \\ Qiongzhou University \\ Sanya, China \\ feng_h007@126.com \\ Jianchun Guo \\ College of tourism management, \\ QiongZhou University \\ Sanya, China \\ gjc92@163.com
}

\author{
Hongxu Wang \\ College of tourism management \\ Qiongzhou University \\ Sanya, China \\ whx16233@126.com
}

\author{
Fujin Zhang \\ College of Electronic Information Engineering \\ QiongZhou University \\ Sanya, China \\ zfj56801@163.com
}

\begin{abstract}
In this essay, we propose a method of forecasting enrollments based on fuzzy time series which is improved by Saxena, Sharma\& Easo. Some sections are improved, Such as the setting of universe of discourse, construction of fuzzy set and Inverse fuzzy number, and forecasting formula. We still used the 22 years freshmen's enrollments data of the University of Alabama to illustrate the forecasting process. The result shows that proposed method provide the smallest AFER and MSE.
\end{abstract}

Keywords-fuzzy set;fuzzy time series; forecasting; percentage; Inverse fuzzy number

\section{INTRODUCTION}

Time series forecasting is widespread used in classical mathematics.Many scholars have acquired great achievements in every field by using Time series forecasting. For example some scholars research Stock Market [3] [5], some research fuzzy data mining for time-series data [6] [8], Li H, Kozma R. who research A dynamic neural network method for time series prediction using the KIII model[9] and so on. Zadeh (1965) publish creativity essay of Fuzzy sets. It may play an important role in research the uncertain fuzzy time series' theory because forecasting problems have some uncertainty. Song and Chissom (1993) propose fuzzy time series and its models and Fuzzy forecasting method have been used to model enrollment data for the University of Alabama[11][12]. Moreover, Song and Chissom(1994) come up with fuzzy time series and its models, Average Forecasting Error Rate (AFER) and Mean Square Error (MSE) to reduce in some extent. Saxena, Sharma and Easo(2012) put forward forecasting formula and used in the same case, as a result, AFER and MSE also reducing. Fuzzy time series forecasting methods are proposed in the literature [14]-[18] and applied to the same case, the results are different. Based on the above work, we propose to modify Saxena, Sharma and Easo's forecasting formula (2012) and gain the new forecasting formula, applied to the same case, making AFER and MSE become the smallest method in the existing thesis.

\section{SOME BASIC KNOWLEDGE}

In this paper, we use some basic concepts in literature[1] and literature[14].

\section{THE MOdified METHOD OF SAXENA, SHARMA AND Easo [1] Proposed Forecasting METHOd Based ON FUZZY TIME SERIES.}

Combined with freshmen's enrollments data of the University of Alabama, proposed the modified method of Saxena, Sharma and Easo [1] come up with forecasting method based on fuzzy time series(A forecasting method of fuzzy time series). Its steps can be drawn as follow:

\section{A. Listed the historical freshmen's enrollments data of university of Alabama}

Song and Chissom at first show the historical freshmen's enrollments data of university of Alabama as table1.

B. Calculated the percentage of year by year change rate of historical data

Calculate the percentage of year by year change of freshmen's enrollments data of university of Alabama.This matter is done by Saxena and Sharma and Easo [1], and fill in table1.

\section{Established discrete universe of discourse}

The percentage of year by year change of freshmen's enrollments data of university of Alabama from 1971 to 1992 as the elements, established discrete universe of discourse. The element in universe of discourse is the percentage of year by year change's historical data from small to large to arrange, established discrete universe of discourse as follow and fill in table1. 


$$
\begin{aligned}
& B=\left\{b_{1972}=3.89, b_{1973}=2.24, b_{1974}=5.98, b_{1975}=5.20, b_{1976}=-0.96, b_{1977}=1.91,\right. \\
& b_{1978}=1.65, b_{1979}=5.96, b_{1980}=0.67, b_{1981}=-3.14, b_{1982}=-5.83, b_{1983}=0.41, \\
& b_{1984}=-2.27, b_{1985}=0.12, b_{1986}=5.41, b_{1987}=5.47, b_{1988}=7.66, b_{1989}=4.52, \\
& \left.b_{1990}=1.89, b_{1991}=0.05, b_{1992}=-2.38\right\} .
\end{aligned}
$$

Table 1 The Historical Enrollments’ Data of University of Alabama, Percentage of Year by Year Change , Element（ [1], [10]）

\begin{tabular}{|c|c|c|c|c|c|c|c|}
\hline year & Enrollments $\mathrm{B}_{\mathrm{i}}$ & percentage & element & year & enrollments $\mathrm{B}_{\mathrm{i}}$ & percentage & element \\
\hline 1971 & 13055 & & & 1982 & 15433 & $-5.83 \%$ & -5.83 \\
\hline 1972 & 13563 & $3.89 \%$ & 3.89 & 1983 & 15497 & $0.41 \%$ & 0.41 \\
\hline 1973 & 13867 & $2.24 \%$ & 2.24 & 1984 & 15145 & $-2.27 \%$ & -2.27 \\
\hline 1974 & 14696 & $5.98 \%$ & 5.98 & 1985 & 15163 & $0.12 \%$ & 0.12 \\
\hline 1975 & 15460 & $5.20 \%$ & 5.20 & 1986 & 15984 & $5.41 \%$ & 5.41 \\
\hline 1976 & 15311 & $-0.96 \%$ & -0.96 & 1987 & 16859 & $5.47 \%$ & 5.47 \\
\hline 1977 & 15603 & $1.91 \%$ & 1.91 & 1988 & 18150 & $7.66 \%$ & 7.66 \\
\hline 1978 & 15861 & $1.65 \%$ & 1.65 & 1989 & 18970 & $4.52 \%$ & 4.52 \\
\hline 1979 & 16807 & $5.96 \%$ & 5.96 & 1990 & 19328 & $1.89 \%$ & 1.89 \\
\hline 1980 & 16919 & $0.67 \%$ & 0.67 & 1991 & 19337 & $0.05 \%$ & 0.05 \\
\hline 1981 & 16388 & $-3.14 \%$ & -3.14 & 1992 & 18876 & $-2.38 \%$ & -2.38 \\
\hline
\end{tabular}

D. Established Inverse fuzzy number of continuous years on discrete universe of discourse $B$

Here are an inverse fuzzy number of consecutive years of normal distribution as follows:

$$
v_{i}=\left\{\begin{array}{l}
\frac{1+0.0002}{\frac{1}{\delta_{1972}}+\frac{0.0002}{\delta_{1973}},} \quad i=1972 \\
\frac{0.0002+1+0.0002}{\frac{0.0002}{\delta_{i-1}}+\frac{1}{\delta_{i}}+\frac{0.0002}{\delta_{i+1}}, \quad 1973 \leq i \leq 1991,} \\
\frac{0.0002+1}{\frac{0.0002}{\delta_{1991}}+\frac{1}{\delta_{1992}}}, \quad i=1992 .
\end{array}\right.
$$

\section{E. Established forecasting formula}

Applied inverse fuzzy number formula (1) to establish the forecasting formula as follows:

$$
F_{i}=B_{i-1} \times \lambda_{i}=B_{i-1} \times\left(1+v_{i} \%\right)
$$

\section{F. Forecasting the freshmen's enrollments data of} university of Alabama from 1971 to 1992

Applied formula (1) and (2) for freshmen's enrollments data of university of Alabama from 1971 to 1972 to imulate forecasting. It can get the forecasting value of freshmen's enrollments data of university of Alabama from 1971 to 1992 and fill in table2.

TABle II Forecasting VALUe of New Method

\begin{tabular}{|c|c|c|c|c|c|c|c|}
\hline Year & Enrolments $\mathrm{B}_{\mathrm{i}}$ & $\delta_{i}(\%)$ & $\lambda_{i}=1+\delta_{x} \%$ & Forecasting value $F_{i}$ & $B_{i}-F_{i}$ & $\left(B_{i}-F_{i}\right)^{2}$ & $\left|B_{i}-F_{i}\right| / A_{i}$ \\
\hline 1971 & 13055 & & & & & & \\
\hline 1972 & 13563 & 3.89 & 1.038894 & 13563 & 0 & 0 & 0.000000 \\
\hline 1973 & 13867 & 2.24 & 1.022422 & 13867 & 0 & 0 & 0.000000 \\
\hline 1974 & 14696 & 5.98 & 1.059779 & 14696 & 0 & 0 & 0.000000 \\
\hline 1975 & 15460 & 5.20 & 1.052068 & 15461 & -1 & 1 & 0.000065 \\
\hline 1976 & 15311 & -0.96 & 0.990395 & 15312 & -1 & 1 & 0.000065 \\
\hline 1977 & 15603 & 1.91 & 1.911082 & 15604 & -1 & 1 & 0.000064 \\
\hline 1978 & 15861 & 1.65 & 1.016503 & 15860 & 1 & 1 & 0.000063 \\
\hline 1979 & 16807 & 5.96 & 1.059475 & 16804 & 3 & 9 & 0.000173 \\
\hline 1980 & 16919 & 0.67 & 1.006703 & 16920 & -1 & 1 & 0.000059 \\
\hline 1981 & 16388 & -3.14 & 0.968561 & 16387 & 1 & 1 & 0.000061 \\
\hline 1982 & 15433 & -5.83 & 0.941532 & 15430 & 3 & 9 & 0.000194 \\
\hline 1983 & 15497 & 0.41 & 1.004102 & 15496 & 1 & 1 & 0.000065 \\
\hline 1984 & 15145 & -2.27 & 0.977179 & 15143 & 2 & 4 & 0.000132 \\
\hline 1985 & 15163 & 0.12 & 1.001200 & 15163 & 0 & 0 & 0.000000 \\
\hline 1986 & 15984 & 5.14 & 1.049725 & 15976 & 8 & 64 & 0.000501 \\
\hline 1987 & 16859 & 5.47 & 1.054693 & 16858 & 1 & 1 & 0.000059 \\
\hline 1988 & 18150 & 7.66 & 1.076583 & 18150 & 0 & 0 & 0.000000 \\
\hline 1989 & 18970 & 4.52 & 1.045429 & 18974 & -4 & 16 & 0.000211 \\
\hline
\end{tabular}




\begin{tabular}{|c|c|c|c|c|c|c|c|}
\hline 1990 & 19328 & 1.89 & 1.018784 & 19326 & 2 & 4 & 0.000103 \\
\hline 1991 & 19337 & 0.05 & 1.000501 & 19338 & -1 & 1 & 0.000052 \\
\hline 1992 & 18876 & -2.38 & 0.975966 & 18872 & 4 & 16 & 0.000212 \\
\hline AFER & & & & & & & \\
\hline MSE & & & & & & 7 \\
\hline
\end{tabular}

G. Testing the forecasting value of freshmen's enrollments data of university of Alabama from 1971 to 1972

Calculated AFER and MSE, fill in table 2.

\section{A COMPARISON OF DIFFERENT FORECASTING MODELS}

Applied this paper's method and different models in literature[1], [2], [14], [4], [15], [16], [17],We research the forecasting enrollments of University of Alabama. The result shown in table3 (many concepts come from table6 in [1]) ,the better evaluate are Saxena, Sharma \& Easo propose the forecasting models in [1] and Stevenson Porter propose forecasting models in[2] \& this paper.

TABLE III COMPARISON OF DifFerent ForeCASTING MODELS [1]

\begin{tabular}{|c|c|c|c|c|c|c|c|c|c|}
\hline Year & $\begin{array}{l}\text { Enroll- } \\
\text { ments }\end{array}$ & $\begin{array}{l}\text { This } \\
\text { paper's } \\
\text { method }\end{array}$ & $\begin{array}{l}\text { Saxena } \\
\text { Sharma } \\
\text { and Easo[1] }\end{array}$ & $\begin{array}{l}\text { Stevenson } \\
\text { Porter[2] }\end{array}$ & $\begin{array}{l}\text { Jilani } \\
\text { Burney and } \\
\text { Ardil[14] }\end{array}$ & $\begin{array}{l}\text { Chen } \\
\text { [15] }\end{array}$ & $\begin{array}{l}\text { Hwang } \\
\text { Chen and } \\
\text { Lee[16] }\end{array}$ & $\begin{array}{l}\text { Chen } \\
\text { [17] }\end{array}$ & $\begin{array}{l}\text { Song and } \\
\text { Chssiom[11] }\end{array}$ \\
\hline 1971 & 13055 & - & - & - & 13579 & - & - & - & - \\
\hline 1972 & 13563 & 13563 & 13486 & 13410 & 13798 & - & - & 14000 & - \\
\hline 1973 & 13867 & 13867 & 13896 & 13932 & 13798 & - & - & 14000 & - \\
\hline 1974 & 14696 & 14696 & 14698 & 14664 & 14452 & 14500 & - & 14000 & - \\
\hline 1975 & 15460 & 15461 & 15454 & 15423 & 15373 & 15500 & - & 15500 & 14700 \\
\hline 1976 & 15311 & 15312 & 15595 & 15847 & 15373 & 15500 & 16260 & 16000 & 14800 \\
\hline 1977 & 15603 & 15604 & 15600 & 15580 & 15623 & 15500 & 15511 & 16000 & 15400 \\
\hline 1978 & 15861 & 15860 & 15844 & 15877 & 15883 & 15500 & 16003 & 16000 & 15500 \\
\hline 1979 & 16807 & 16804 & 16811 & 16773 & 17079 & 16500 & 16261 & 16000 & 15500 \\
\hline 1980 & 16919 & 16920 & 16916 & 16897 & 17079 & 16500 & 17407 & 16833 & 16800 \\
\hline 1981 & 16388 & 16387 & 16425 & 16341 & 16497 & 16500 & 17119 & 16833 & 16200 \\
\hline 1982 & 15433 & 15430 & 15657 & 15671 & 15737 & 15500 & 16188 & 16833 & 16400 \\
\hline 1983 & 15497 & 15496 & 15480 & 15507 & 15737 & 15500 & 14833 & 16000 & 16800 \\
\hline 1984 & 15145 & 15143 & 15214 & 15200 & 15024 & 15500 & 15497 & 16000 & 16400 \\
\hline 1985 & 15163 & 15163 & 15184 & 15218 & 15024 & 15500 & 14745 & 16000 & 15500 \\
\hline 1986 & 15984 & 15976 & 15995 & 16035 & 15833 & 15500 & 15163 & 16000 & 15500 \\
\hline 1987 & 16859 & 16858 & 16861 & 16903 & 17079 & 16500 & 16384 & 16000 & 15500 \\
\hline 1988 & 18150 & 18150 & 17965 & 17953 & 17991 & 18500 & 17659 & 16833 & 16800 \\
\hline 1989 & 18970 & 18974 & 18964 & 18879 & 18802 & 18500 & 19150 & 19000 & 19300 \\
\hline 1990 & 19328 & 19326 & 19329 & 19303 & 18994 & 19500 & 19770 & 19000 & 17800 \\
\hline 1991 & 19337 & 19338 & 19378 & 19432 & 18994 & 19500 & 19928 & 19000 & 19300 \\
\hline 1992 & 18876 & 18872 & 18984 & 18966 & 18916 & 18500 & 15837 & 19000 & 19600 \\
\hline AFER & & $0.0099 \%$ & $0.34 \%$ & $0.57 \%$ & $1.02 \%$ & $1.52 \%$ & $2.44 \%$ & $3.11 \%$ & $3.11 \%$ \\
\hline MSE & & 7 & 9169 & 21575 & 41426 & 86696 & 226611 & 321418 & 407507 \\
\hline
\end{tabular}




\section{CONCLUSION}

When Song and Chissom proposed the first forecasting model of fuzzy time series in1993 [11], it is symbol that the theory of fuzzy time series have already been born. Up to now, many literatures and researchers research and develop this theory. Such as literature[1], [2], [3], [11], [12], [13], [14], [15], [16], [17], [18]. This paper modifies the above research and proposed a new forecasting method of fuzzy time series. Thus, this method further reduces AFER and MSE. From the table3, we can see that this forecasting method's AFER and MSE less than existing method.

\section{REFERENCES}

[1] Preetika Saxena, Kalyani Sharma, Santhosh Easo. Foreeca enrollment based on fuzzy time series with higher forecast accuracy rate. International Journal of Computer Technology and Applications, 2012, vol.3. No.3.pp.957-961

[2] Stevension Porter. Fuzzy time series forecasting using percentage change as the universe of discourse. Proceedings of World Academy of Science. Engineering and Technology. 2009, vol.55, pp.154-157

[3] Fu T-C, Chung F-L, Luk R, Ng C-M. Stock time series pattern matching: Template-based vs. rule-based approaches. Engineering Applications of Artificial Intelligence 2007, 20: 344-364

[4] Chen T-L, Cheng C-H, Teoh H-J, High-order fuzzy time-series based on multi-period adaptation model for forecasting stock markets. Physica A, 2008, 387: 876-888

[5] Chu H-H, Chen T-L, Cheng C-H, Huang C-C. Fuzzy dual-factor time-service for stock index forecasting. Expert Systems with Application, 2009, 36: 165-171
[6] Hu X, Xu P, Wu S Z, Asgari S, Bergsneider M. A data mining framework for time series estimation. Journal of Biomedical Informatics, 2010, 43: 190-199

[7] Fu T-C. A review on time series data mining. Engineering Applications of Artificial Intelligence, 2011, 24: 164-181

[8] Chen C-H, hong T-P, Tseng V S. fuzzy data mining for time-series data. Applied Soft Computing, 2012, 12: 536-542

[9] Li H, Kozma R. A dynamic neural network method for time series prediction using the KIII model. Proceedings of the 2003 International Joint Conference on Neural Networks, 1: 347-352

[10] Zadeh L A. Fuzzy sets [J]. Information and Control, 1965, (8): 338353

[11] Song Q, Chissom B S. Forecasting enrollments with fuzzy time series--part 1 [J]. Fuzzy Set and Systems, 1993, 54: 1-9

[12] Song Q, Chissom B S. Fuzzy time series and its models [J]. Fuzzy Set and Systems, 1993, 54:169-277

[13] Song Q, Chissom B S.Forecasting enrollments with fuzzy time series: Part II. Fuzzy Sets and Systems, 1994, Vol.62, pp.1-8

[14] T A Jilani, S M A Burney, C Ardil. Fuzzy metric approach for fuzzy time series forecasting based on frequency density based partitioning. Proceedings of Wold Academy of Science, Engineering and Technology, 2007, vol. 34, pp. 333-338

[15] S M Chen. Forecasting enrollments based on high-order fuzzy time series. Cybernetics and Systems: An International Journal, 2002, vol. 33. pp. 1-16

[16] J R Hwang, S M Chen, C H Lee. Handling forecasting problems using fuzzy time series. Fuzzy Sets and Systems, 1998, vol.100, pp. 217-228

[17] S M Chen. Forecasting enrollments based on fuzzy time series. Fuzzy Sets and Systems, 1996, vol.81, pp. 311-319

[18] S. M. Chen and C. C. Hsu. A new method to forecasting enrollments using fuzzy time series. International Journal of Applied Science and Engineering. 2004, vol.2, no 3, pp.234-244 